\title{
Epidémiologie de la theilériose tropicale bovine (infection par Theileria annulata) en Tunisie : une synthèse
}

\author{
M. Gharbi ${ }^{1} *$ M.R. Rjeibi ${ }^{1}$ M.A. Darghouth ${ }^{1}$
}

\begin{abstract}
Mots-clés
Bovin - Theilériose tropicale bovine Epidémiologie - Lutte contre les maladies - Tunisie.
\end{abstract}

\begin{abstract}
Résumé
Le présent article est une revue bibliographique de l'épidémiologie de la theilériose tropicale bovine en Tunisie. C'est une parasitose spécifique due à la présence et à la multiplication dans les phagocytes mononucléés puis dans les érythrocytes d'un protozoaire de la famille des Theileridae, Theileria annulata. Elle est transmise de manière biologique par plusieurs espèces de tiques de la famille des Ixodidae, appartenant au genre Hyalomma. L'implication de trois acteurs très éloignés sur le plan taxonomique est à l'origine d'une maladie dont l'épidémiologie est très complexe. Cette infection évolue selon trois modes enzootiques : (a) l'enzootie stable qui résulte d'un état d'équilibre entre l'hôte et le parasite, (b) l'enzootie instable modérée qui est due à la présence $d^{\prime}$ une faible population de tiques engendrant des cas cliniques chez des animaux âgés de deux à trois ans, et (c) I'enzootie instable élevée dans laquelle la population de tiques est tellement faible que la probabilité de rencontre entre une tique infectée et un hôte sensible est minime. Le type de situation épidémiologique dans lequel se trouve l'élevage permet de planifier un programme de lutte adapté.
\end{abstract}

\section{INTRODUCTION}

D'après le ministère de l'Agriculture tunisien (27), la population bovine du pays est estimée à 606400 bovins. Le secteur de l'élevage connaît depuis des années plusieurs types de contraintes : (a) structurelles, 45 p. 100 des bovins appartenant à des éleveurs qui possèdent au plus dix hectares et dont la majorité des locaux d'élevage sont rudimentaires ; (b) financières, ces petits éleveurs ayant une trésorerie assez souvent déséquilibrée ; (c) humaines, la majorité des éleveurs de bovins n'ayant qu'un niveau d'instruction primaire ; (d) nutritionnelles, les aliments concentrés pour bovins étant importés, ils sont soumis aux aléas de la disponibilité et du prix du marché ; (e) climatiques, les deux tiers de la superficie du pays étant arides ou sahariens ; et (f) sanitaires, certaines maladies comme la brucellose, les boiteries, les réticulo-péritonites par corps étrangers et les mammites demeurant fréquentes en Tunisie.

1. Laboratoire de parasitologie, Ecole nationale de médecine vétérinaire, Univ. Manouba, 2020 Sidi Thabet, Tunisie.

* Auteur pour la correspondance

E-mail : gharbim2000@yahoo.fr
La theilériose tropicale représente une contrainte majeure au développement de l'élevage au Maghreb (28). Ce problème a été mis en évidence par Sergent et coll. (30) dès le début du $\mathrm{XX}^{\mathrm{e}}$ siècle, si bien qu'un groupement d'éleveurs algériens a financé les travaux de recherche de cette équipe pendant plusieurs années. En Tunisie, au moins 2500 cas cliniques de theilériose tropicale nécessitent un traitement chaque année (1). Il faut y ajouter les cas non diagnostiqués et non pris en charge, dont la plupart sont mortels puisque, même chez les animaux traités, le taux de létalité a été estimé entre 10 et 13,5 p. $100(4,21)$. La theilériose tropicale bovine est à l'origine d'une diminution du poids des animaux, d'une hypogalaxie qui peut aller dans certains cas jusqu'à l'agalaxie, d'avortements et, dans certains cas, de mortalités $(12,13)$. De plus, le coût du traitement est très élevé puisqu'une dose de buparvaquone coûte environ $30 €$ et qu'il faut y associer un traitement symptomatique. Enfin, le portage asymptomatique de T. annulata, qui atteint dans les élevages en situation d'enzootie stable 100 p. 100 des animaux, est à l'origine de pertes en gain moyen quotidien qui ont été estimées à 15 p. 100 du poids des veaux infectés (12). Cet état de portage asymptomatique est également à l'origine d'une diminution de la production laitière de 0,77 litre par jour et par vache infectée (article accepté, Asian Pac. J. Trop. Dis.). 
Tous ces éléments motivent la mise en place d'un programme de lutte qui ne peut réussir que s'il est adapté au contexte épidémiologique de l'élevage. La confrontation d'éléments épidémiologiques aux éléments cliniques aide par ailleurs les cliniciens à établir le diagnostic de la theilériose tropicale bovine (14). Dans cette revue bibliographique, nous proposons une étude de l'épidémiologie de la theilériose tropicale bovine en Tunisie, épidémiologie très complexe car elle fait intervenir trois acteurs : le bovin (hôte intermédiaire), la tique du genre Hyalomma (hôte définitif) et le protozoaire (figure 1). L'interaction entre ces trois acteurs est régie par plusieurs facteurs biotiques et abiotiques détaillés ci-dessous.

\section{- EPIDEMIOLOGIE DESCRIPTIVE}

La theilériose tropicale bovine est une maladie qui affecte les bovins sur trois continents : l'Europe (l'Europe du Sud), l'Afrique (la Mauritanie, l'Afrique du Nord et le Soudan) et l'Asie (de la côte méditerranéenne à la Chine). Cette large distribution géographique est à l'origine d'un polymorphisme épidémiologique en relation avec (a) la variété des vecteurs - plusieurs espèces de tiques du genre Hyalomma, comme $H$. scupense, $H$. lusitanicum, $H$. anatolicum, $H$. dromedarii étant des vecteurs de $T$. annulata -, (b) les races de bovins et (c) les conditions abiotiques. Cette complexité a été confirmée par la mise en place d'un indicateur de présence d'infections multiples (multiplicity of infection [MOI]) dont le calcul a permis de montrer la présence d'un polymorphisme génétique de $T$. annulata beaucoup plus important en Turquie qu'en Tunisie (35). Ce polymorphisme a été objectivé par l'étude de séquences microsatellites non soumises à une pression de sélection. Un bovin peut ainsi être coïnfecté par plusieurs génotypes (2, 35) mais il est impossible de préciser le nombre d'infections qu'il a subi. Le nombre moyen d'allèles par bovin varie entre deux et quatre mais seules dix séquences microsatellites ont été utilisées dans l'étude de Weir et coll. (35).

En Tunisie, la theilériose tropicale bovine est une maladie qui évolue durant la période chaude (été) ; elle est considérée comme la principale maladie estivale des bovins. Cette typologie saisonnière de la maladie est en relation avec la dynamique d'activité de la tique vectrice. Bouattour et coll. (5) ont ainsi montré que le pic d'incidence clinique de la theilériose tropicale survient en moyenne 15 jours après le pic d'infestation par les adultes de $H$. scupense (syn. $H$. detritum). La maladie est rapportée entre mai et septembre avec un pic d'incidence en juillet-août $(8,9)$. Ainsi, 87 p. 100 des

Figure 1 : cycle biologique de la theilériose tropicale bovine en Tunisie. cas cliniques de theilériose tropicale recensés entre 1987 et 2007 $(n=2507)$ ont été diagnostiqués en été (de juin à août) par le laboratoire de parasitologie de l'Ecole nationale de médecine vétérinaire de Sidi Thabet (11) (figure 2). Des cas de rechutes consécutives à un déséquilibre de la relation hôte-parasite peuvent toutefois avoir lieu à n'importe quel moment de l'année et donner des cas cliniques qui peuvent être sévères. Ainsi, lors de l'étude précédemment mentionnée, 1 p. 100 des cas cliniques de theilériose tropicale avaient été rapportés en hiver (entre décembre et février) alors que les tiques vectrices sont en hibernation (11) (figure 2).

La distribution annuelle de la maladie est très différente dans le biotope oasien de Gabès (sud-est de la Tunisie) où les cas cliniques sont rapportés entre août et octobre-novembre, le pic se situant vers le mois de septembre (19). Ce décalage serait dû à l'impact du climat oasien chaud et humide qui occasionnerait un déplacement de la saison d'activité des tiques dans le temps et provoquerait une entrée plus tardive des nymphes gorgées en hibernation.

Ces exemples montrent l'importance des facteurs abiotiques dans la typologie épidémiologique de la theilériose tropicale bovine. La dynamique d'activité de l'espèce vectrice est également très importante pour expliquer la survenue de la maladie. Dans certains pays comme le Soudan, la tique vectrice, en l'occurrence Hyalomma anatolicum, est active en permanence et il n'y a donc pas de saison particulière de transmission, les cas cliniques étant observés tout au long de l'année (29). La typologie épidémiologique conditionne l'équilibre entre l'hôte et le parasite. Lorsque la transmission par les tiques est continue dans le temps, la pression d'infection est permanente et généralement élevée, ce qui crée une situation d'enzootie stable dans les élevages. Dans ce contexte épidémiologique particulier, la lutte est plus difficile à mener du fait de la présence continue de tiques vectrices. Le choix de la stratégie de lutte à conduire doit être effectué après des études coût-bénéfice car les pertes financières en lait et en viande sont faibles et imperceptibles par les éleveurs, mais persistantes dans le temps (12).

En Tunisie, la majorité des cas cliniques de theilériose tropicale est observée dans les étages bioclimatiques humide et subhumide, d'une part, et semi-aride, d'autre part, où $H$. scupense est la tique la plus commune $(3,4,9)$ (figures 3 et 4$)$. Ces deux zones bioclimatiques concentrent d'ailleurs la majorité du cheptel bovin tunisien (respectivement 39 et 41 p. 100). La présence au nord de la Tunisie de plusieurs espèces de tiques vectrices de Babesia spp. (Ixodes ricinus, Hyalomma marginatum, Rhipicephalus annulatus) est de plus à l'origine de coïnfections cliniques. Touhami (32)

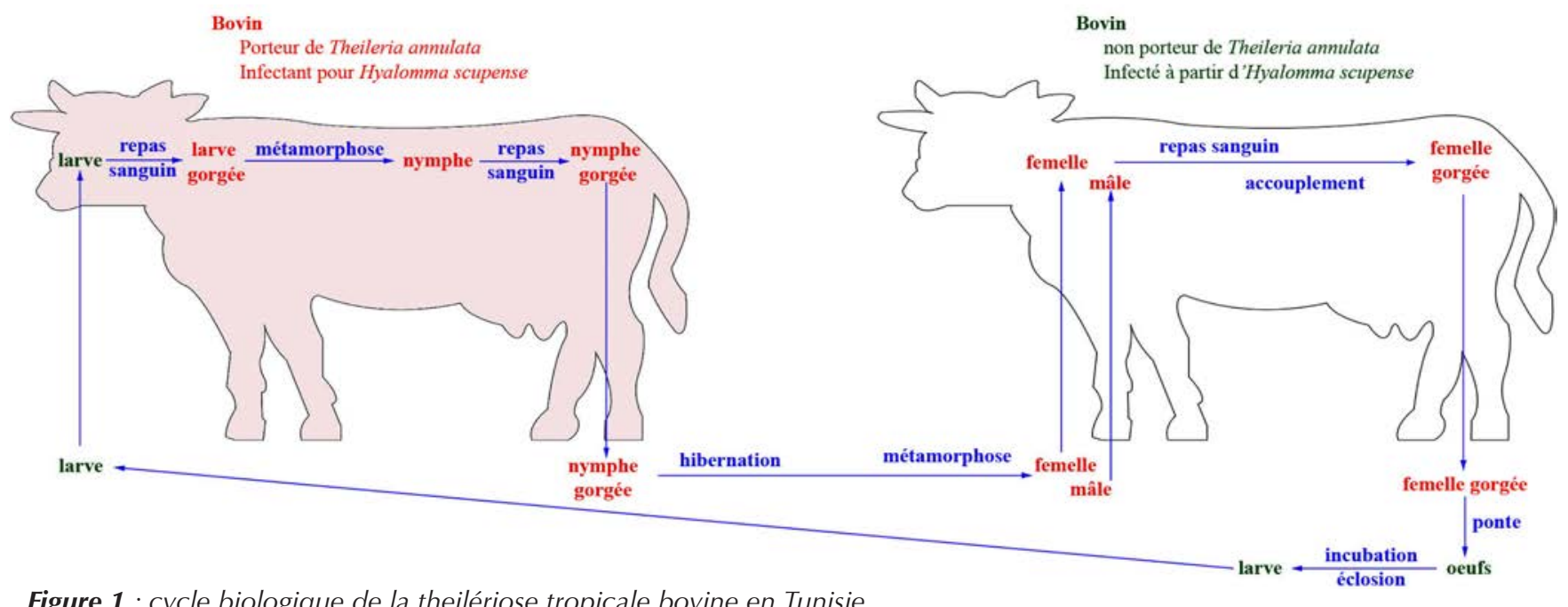




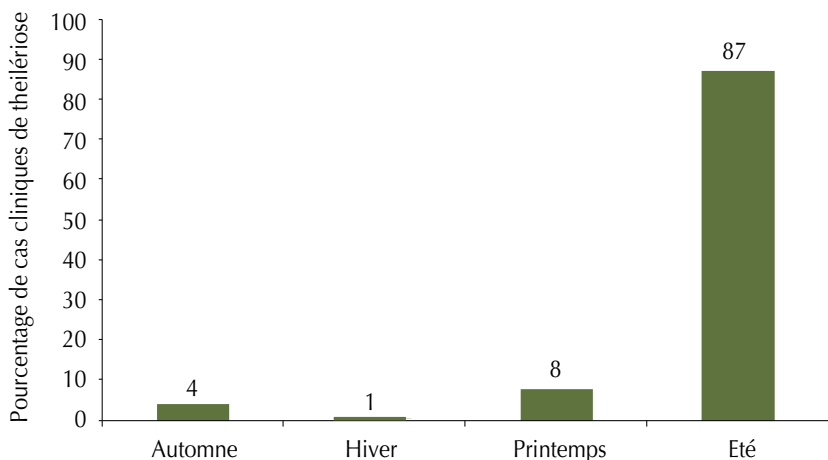

Figure 2 : distribution saisonnière de la theilériose tropicale bovine dans le nord de la Tunisie entre 1987 et 2007 (d'après Fatnassi, 2010).
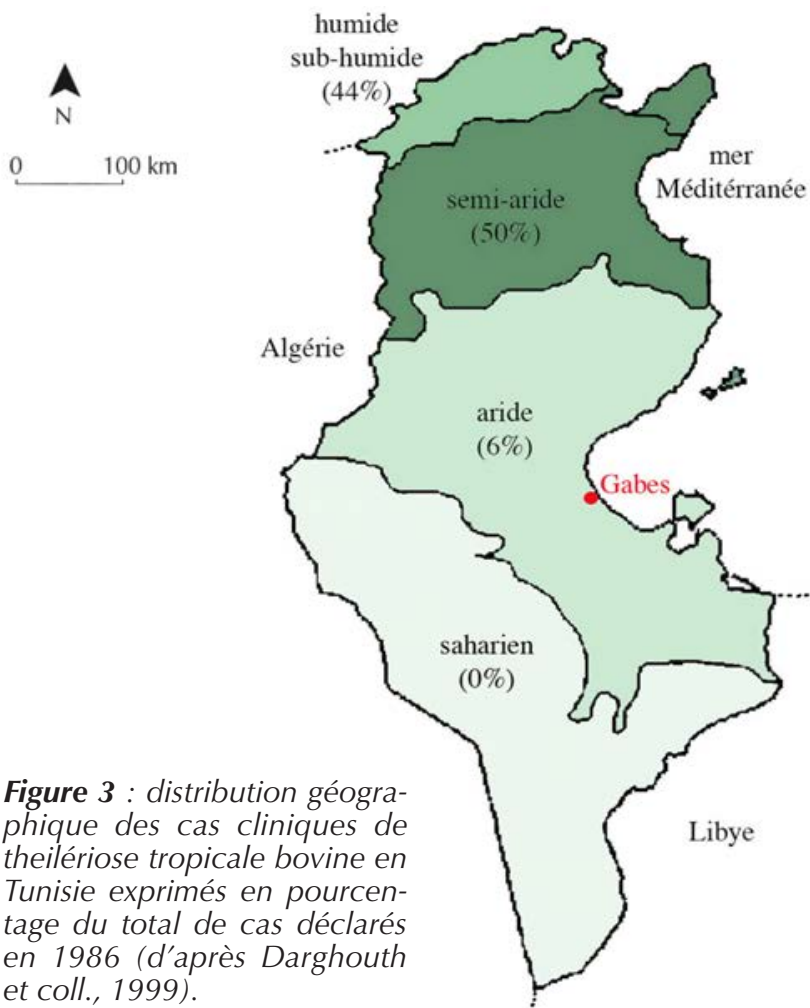

Figure 3 : distribution géographique des cas cliniques de theilériose tropicale bovine en Tunisie exprimés en pourcentage du total de cas déclarés en 1986 (d'après Darghouth et coll., 1999).

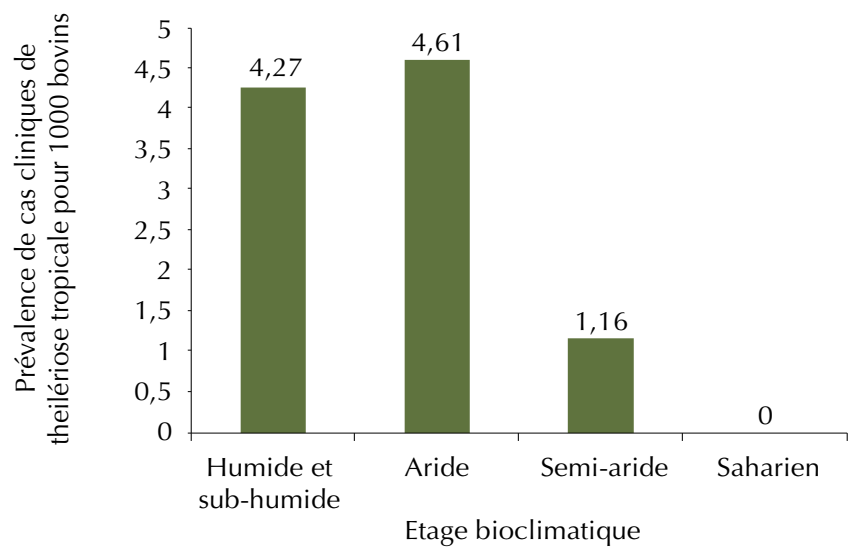

Figure 4 : nombre de cas cliniques de theilériose tropicale bovine par rapport à la population bovine dans les différents étages bioclimatiques tunisiens ( $d$ 'après Darghouth et col., 1999, et le ministère de l'Agriculture, 2006). a ainsi montré que 20 p. 100 des cas cliniques de piroplasmoses étaient des coïnfections par T. annulata et Babesia spp. Enfin, quelques rares animaux (2 p. 100) étaient infectés par trois hémopathogènes (T. annulata, Babesia sp. et Anaplasma marginale). Ces associations sont déroutantes pour les cliniciens qui généralement ne suspectent qu'une seule maladie.

L'étage bioclimatique aride ne concentre que 6 p. 100 des cas cliniques alors qu'il contient 19 p. 100 du cheptel bovin tunisien. Dans l'étage bioclimatique saharien, qui ne regroupe que 3 p. 100 $\mathrm{du}$ cheptel bovin national, la theilériose tropicale est absente du fait de l'absence totale du vecteur (figures 3 et 4).

\section{EPIDEMIOLOGIE ANALYTIQUE}

\section{Sources du parasite}

En Tunisie, les sources directes du parasite sont représentées par les adultes d'une seule espèce de tiques, Hyalomma scupense. Dans d'autres pays, $H$. anatolicum, $H$. lusitanicum et $H$. dromedarii sont les vecteurs du protozoaire (34). Ils transmettent l'infection à l'occasion d'un repas sanguin. Les sources indirectes sont représentées par les bovins infectés par T. annulata, et notamment par les animaux porteurs asymptomatiques (et à un degré moindre par les animaux malades) qui infectent les larves et les nymphes de tiques à l'occasion du repas sanguin.

\section{Mode de transmission}

La transmission de $T$. annulata est assurée par la salive de la tique adulte, les parasites étant injectés au stade sporozoïte dès le troisième jour du repas sanguin. La transmission par des seringues contaminées est possible mais son rôle dans l'épidémiologie de la theilériose tropicale est accessoire.

\section{Réceptivité}

Les facteurs espèce, race et âge interviennent dans la réceptivité des animaux à l'infection par T. annulata.

\section{Espèce}

Certains bovinés peuvent être infectés par $T$. annulata, notamment le bovin (Bos taurus) et le buffle asiatique (Bubalus bubalis), mais ils sont sensibles à la maladie à des degrés différents. Chez ce dernier, on observe des symptômes qui sont rarement observés chez le bœuf, comme la pneumonie, ou qui ne le sont jamais, comme l'œdème de la cornée qui aboutit fréquemment à la cécité (25).

\section{Race}

Glass et coll. (18) ont montré que les veaux zébus de race Sahiwal (Bos indicus) exprimaient un tableau clinique significativement moins sévère que les veaux de race Holstein (Bos taurus). Les veaux Sahiwal présentent un accès fébrile plus court (2-3 jours en moyenne contre 7 jours chez les bovins Holstein) et montrent des concentrations significativement plus faibles et plus fugaces d'une protéine de la phase aiguë (acide alpha 1 glycoprotéine).

Les races bovines autochtones sont habituellement plus résistantes, seuls quelques sujets développant une forme atténuée de la maladie. En revanche, les races améliorées comme la Frisonne Pie noire, la Holstein ou les produits de leurs croisements avec les races locales sont plus sensibles. L'effet de la race est clairement illustré par l'observation en Tunisie, dans des étables en situation d'enzootie stable de theilériose, d'une corrélation positive entre l'incidence de la maladie chez les veaux et le pourcentage du génotype Frisonne Pie noire. Ainsi en 1991, l'incidence des cas 
cliniques dans des élevages tunisiens était de 7,7 p. 100 et l'ensemble des veaux présentaient un phénotype local (7) (figure 5). En 1994, 50 p. 100 des veaux exprimaient un phénotype Frison Pie noir : l'incidence des cas cliniques a atteint 21 p. 100 et la séroprévalence pendant l'automne était toujours proche de 100 p. 100 (31). En 2001, l'ensemble des veaux avaient un phénotype Frison Pie noir et l'incidence des cas cliniques a atteint 52 p. 100 (20). Cette augmentation de l'incidence pourrait également être attribuée à une diminution des niveaux d'infestation des animaux par les tiques due à l'intensification des efforts de lutte fournis par les éleveurs, lutte qui aurait réduit les possibilités d'acquisition précoce d'une immunité protectrice. Néanmoins, les trois enquêtes acarologiques effectuées dans ces mêmes élevages entre 1991 et 2007 n'ont pas mis en évidence un changement significatif du nombre de tiques $(5,12,15)$.

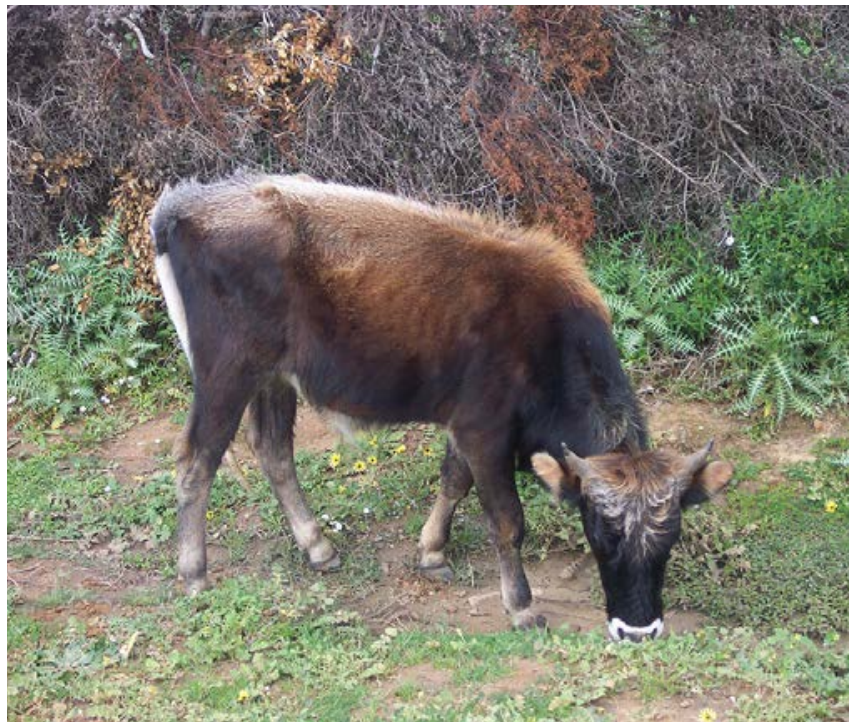

Figure 5 : bovin de race Brune de l'Atlas, race résistante à la theilériose tropicale bovine.

D'après Glass (17), la sensibilité des bovins à la theilériose tropicale est déterminée par la capacité de l'hôte à réguler la réponse immune à l'infection. La meilleure résistance des animaux autochtones serait consécutive à un processus coévolutif extrêmement ancien alors que les races exotiques ne sont pas encore adaptées au protozoaire. Cette grande sensibilité serait d'ailleurs amplifiée par les contraintes de production plus élevée auxquelles les animaux de ces races sont soumis.

\section{Age}

L'âge est un facteur important corrélé à au moins quatre paramètres : (a) la moindre attractivité des veaux pour les tiques, observée par exemple lors d'un suivi d'infestation dans des élevages en situation d'enzootie stable à la theilériose tropicale, au cours duquel les vaches avaient une infestation moyenne de 75,8 tiques/vache/saison alors qu'elle n'était respectivement que de 50,6 et 12,4 tiques/veau/saison chez les veaux mâles et femelles (15) ; (b) l'accumulation d'infections successives car l'analyse génétique des populations de $T$. annulata dans des élevages en situation d'enzootie stable a montré la présence d'un état de panmixie parmi les isolats aussi bien turcs que tunisiens (35) ; (c) la présence d'anticorps colostraux; et (d) la tolérance des veaux visà-vis des hémoparasites, phénomène observé lors d'infection par d'autres hémopathogènes.

\section{Facteurs favorisants}

Trois facteurs favorisent le développement de la maladie : le mode d'élevage, l'état de l'étable et les conditions climatiques.

\section{Mode d'élevage}

Comme la tique vectrice $H$. scupense est endophile, les animaux élevés en plein air sont moins exposés à l'infection que ceux vivant dans des étables. D'ailleurs, Sergent et coll. (30) avaient déjà recommandé d'éloigner les bovins des étables pendant toute la période estivale pour diminuer l'incidence de la theilériose tropicale. Dans d'autres régions du monde, comme la péninsule ibérique où le vecteur $H$. lusitanicum est exophile, le pâturage représente au contraire un important facteur de risque (33).

\section{Etat de l'étable}

Les étables mal conçues et mal entretenues, dont les murs présentent des crevasses et des fissures et où l'on note la présence de tas de bouses (utilisées par les éleveurs comme combustible dans des fours traditionnels), offrent des gîtes favorables à la ponte des femelles et à l'hibernation des nymphes gorgées de $H$. scupense (figure 6). A l'opposé, les étables bien conçues et bien entretenues, dont les crépis muraux sont lisses, sans anfractuosités, n'hébergent généralement pas de tiques. Ainsi, on note, en Tunisie, l'absence de cas cliniques de theilériose tropicale dans les étables modernes.

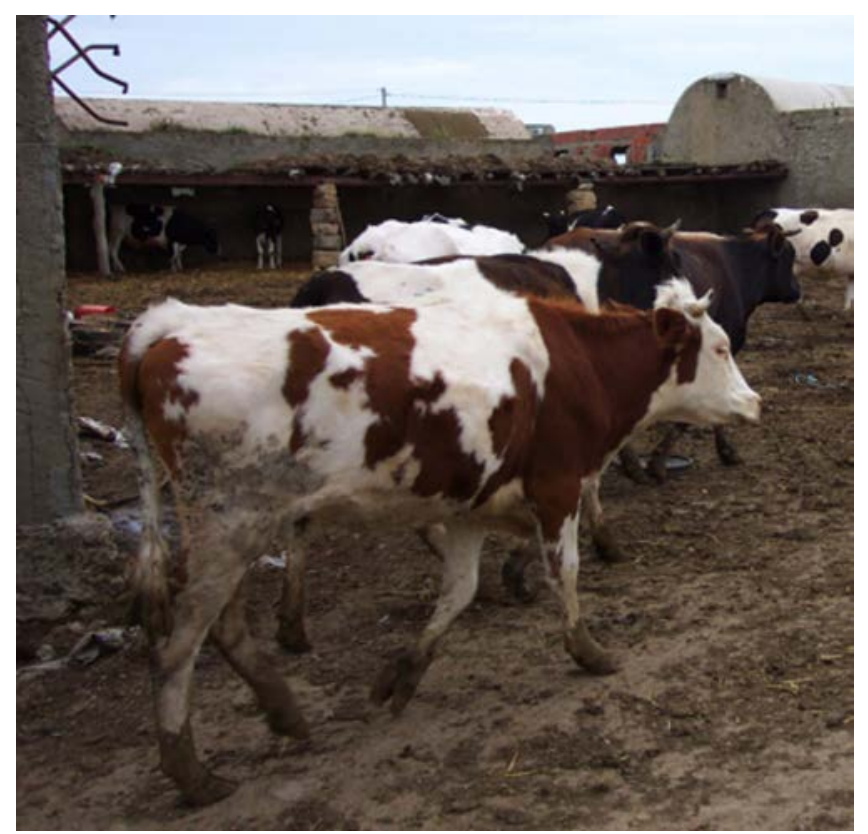

Figure 6 : élevage de bovins métis en situation d'enzootie stable à la theilériose tropicale bovine. Notez l'état des murs qui est un bon indicateur de la présence de Hyalomma scupense.

\section{Conditions climatiques}

Les facteurs climatiques interviennent en conditionnant l'activité de la tique. En Tunisie, les épisodes de sirocco (vent violent, sec et chaud, venant du Sahara) en début d'été entraînent une sortie massive de vagues de tiques de leurs gîtes d'hibernation et, par conséquent, une augmentation différée dans le temps de l'incidence de la theilériose tropicale. Sergent et coll. (30) avaient noté que les cas cliniques de theilériose tropicale étaient plus graves lors de sirocco du fait d'une intensité d'infestation plus élevée par les tiques durant ces périodes. 


\section{EPIDEMIOLOGIE SYNTHETIQUE}

Par analogie avec la typologie épidémiologique de la babésiose à Babesia bovis décrite en Australie (26), Darghouth et coll. (7) ont utilisé trois indicateurs épidémiologiques pour classer les élevages : (a) les séroprévalences avant et après la saison d'activité des tiques adultes, (b) l'incidence des cas cliniques, et (c) le risque relatif de maladie, c'est-à-dire le ratio de l'incidence clinique par rapport à la séroprévalence. Ils ont ainsi identifié deux situations enzootiques en Tunisie : l'enzootie stable et l'enzootie instable (tableau I).

\section{Enzootie stable}

L'état d'enzootie stable est la résultante d'une infection précoce par $T$. annulata de tous les veaux dès leur première saison estivale (correspondant à la première saison de tiques). Comme les veaux sont faiblement infestés par la tique vectrice (3) et qu'ils sont protégés par la tolérance du jeune âge et/ou le colostrum, ils sont progressivement immunisés, durant ce premier été, contre la theilériose tropicale avec de faibles doses infectantes (notion d'effetdose). Les cas cliniques sont virtuellement absents chez les animaux de plus d'une saison estivale qui se sont immunisés dès leur jeune âge. Cette situation enzootique correspond aux élevages de type traditionnel où la population de tiques vectrices est élevée.

D'un point de vue sérologique, l'état d'enzootie stable se caractérise par une séroprévalence automnale de 100 p. 100 chez les veaux après la première saison estivale. Néanmoins, 7,7 p. 100 des veaux développent quand même la maladie (7). Ces cas cliniques seraient dus à une mauvaise prise colostrale, à une infestation simultanée par plusieurs tiques infectantes ou à l'infection par des souches très virulentes. Cette situation épidémiologique est observée dans 3,8 p. 100 des élevages du nord de la Tunisie (10).

\section{Enzootie instable}

Il s'agit d'un état enzootique caractérisé par une population de tiques vectrices insuffisante pour assurer l'infection de tous les veaux en première saison estivale. Il est possible de distinguer deux situations d'enzootie instable : l'enzootie instable modérée et l'enzootie instable élevée.

\section{Enzootie instable modérée}

Les cas cliniques sont constatés chez les bovins jusqu'à l'âge de trois saisons de tiques (c'est à dire trois étés). Les veaux en première saison estivale sont peu affectés à cause de leur faible attractivité pour les tiques. Les animaux s'immunisent pendant les deuxième et troisième étés, pendant lesquels des cas cliniques sont observés. En revanche, les animaux de plus de trois étés sont en totalité naturellement immunisés. L'infestation des bovins adultes par les tiques est modérée, inférieure à 50 tiques/vache/saison. L'enzootie instable modérée est observée chez respectivement 19,6 et 28 p. 100 des élevages dans les étages bioclimatiques semi-aride et subhumide de la Tunisie (9).

\section{Enzootie instable élevée}

La séroprévalence automnale est inférieure à 100 p. 100 dans toutes les catégories d'âge des bovins. Les cas cliniques sont surtout observés chez les animaux de quatre saisons de tiques et plus, c'est-à-dire chez les vaches laitières, du fait de leur effectif important dans les étables à risque et du stress de production (lactation et gravidité) auquel elles sont exposées. Cet état est lié à une faible population de tiques vectrices, l'infestation moyenne étant de trois tiques/vache/saison (22). L'enzootie instable élevée est de loin la situation la plus fréquente en Tunisie, présente dans 76,2 et 72 p. 100 des élevages se trouvant respectivement dans les étages bioclimatiques semi-aride et subhumide (10).

\section{Etat indemne}

L'importation de grands effectifs de génisses gravides de races améliorées (Frisonne Pie noire, Brown Swiss, et Holstein) a débuté en Tunisie dans les années 1970 pour le compte des unions coopératives de production agricole (coopératives qui occupaient pour la majorité d'entre elles des fermes abandonnées par les Français après l'indépendance de la Tunisie). Des épizooties mortelles de theilériose tropicale se sont déclarées dans ces cheptels et ont provoqué d'importantes pertes financières (données non publiées). Du fait de l'absence à cette époque de molécules theiléricides actives,

\section{Tableau}

Etats enzootiques de la theilériose tropicale bovine en Tunisie

\begin{tabular}{|c|c|c|c|c|c|c|}
\hline $\begin{array}{l}\text { Etat } \\
\text { enzootique }\end{array}$ & $\begin{array}{l}\text { Infestation par les } \\
\text { tiques vectrices }\end{array}$ & Types d'élevages & $\begin{array}{l}\text { Incidence de } \\
\text { la maladie }\end{array}$ & $\begin{array}{l}\text { Prévalence } \\
\text { sérologique } \\
\text { automnale }\end{array}$ & $\begin{array}{l}\text { Population à } \\
\text { risque }\end{array}$ & $\begin{array}{l}\text { Fréquence des } \\
\text { élevages en } \\
\text { Tunisie }\end{array}$ \\
\hline $\begin{array}{l}\text { Enzootie } \\
\text { stable }\end{array}$ & $\begin{array}{l}\text { Elevée (> } 50 \text { tiques/ } \\
\text { animal/saison) }\end{array}$ & $\begin{array}{l}\text { Bovins de race locale } \\
\text { Bovins métis } \\
\text { Races exotiques (moins } \\
\text { fréquemment) }\end{array}$ & Faible & $\begin{array}{l}\text { Proche de } \\
100 \% \text { chez tous } \\
\text { les animaux }\end{array}$ & $\begin{array}{l}\text { Veaux en } \\
\text { première saison } \\
\text { estivale } \\
\text { Nouveaux } \\
\text { venus }\end{array}$ & $\begin{array}{l}<5 \% \text { des } \\
\text { élevages }\end{array}$ \\
\hline $\begin{array}{l}\text { Enzootie } \\
\text { instable } \\
\text { modérée }\end{array}$ & $\begin{array}{l}\text { Modérée (10 à } 50 \\
\text { tiques/animal/saison) }\end{array}$ & $\begin{array}{l}\text { Bovins métis } \\
\text { Races exotiques (moins } \\
\text { fréquemment) }\end{array}$ & Modérée & $\begin{array}{l}100 \% \text { à partir } \\
\text { de la troisième } \\
\text { saison estivale }\end{array}$ & $\begin{array}{l}\text { Animaux } \\
\text { jusqu'à trois } \\
\text { étés }\end{array}$ & $\begin{array}{l}19 \text { à } 20 \% \text { des } \\
\text { élevages }\end{array}$ \\
\hline $\begin{array}{l}\text { Enzootie } \\
\text { instable } \\
\text { élevée }\end{array}$ & $\begin{array}{l}\text { Faible }(<5 \text { à } 10 \\
\text { tiques/animal/saison) }\end{array}$ & Races exotiques & $\begin{array}{l}\text { Modérée à } \\
\text { élevée }\end{array}$ & $\begin{array}{l}\text { Faible à } \\
\text { modérée }\end{array}$ & Vaches laitières & $\begin{array}{l}76 \text { à } 81 \% \text { des } \\
\text { élevages }\end{array}$ \\
\hline
\end{tabular}


ces épizooties ont incité les services vétérinaires à réaliser la mise aux normes des étables, mesure qui a abouti à l'éradication de la theilériose dans ces élevages. A partir de cette période, les services vétérinaires tunisiens ont recommandé aux élevages étatiques la construction d'étables modernes constituées d'espaces couverts sans murs.

\section{- EPIDEMIOLOGIE PROSPECTIVE}

Certains facteurs peuvent intervenir pour changer la typologie épidémiologique future de la theilériose tropicale bovine en Tunisie. Nous en citerons deux qui sont d'une grande importance :

- le développement de populations de T. annulata résistantes visà-vis de la buparvaquone. Cette molécule est actuellement le meilleur theiléricide (23). Le premier cas de résistance dans le monde a été identifié chez des vaches d'une ferme du nord de la Tunisie : quatre parmi ces vaches sont mortes de theilériose tropicale malgré deux injections de buparvaquone aux doses recommandées. Cette résistance a été par la suite confirmée sur des cultures cellulaires par Mhadhbi et coll. (24). Le nombre de cas de résistance confirmés au laboratoire de parasitologie de l'Ecole nationale de médecine vétérinaire est en nette augmentation (données non publiées) ; - l'extension de la distribution géographique de $H$. scupense suite aux changements climatiques, qui induirait des modifications similaires de la distribution de la theilériose tropicale bovine. D'autre part, $H$. dromedarii est une tique fréquente au centre et au sud de la Tunisie (16). Si elle effectuait un repas sanguin sur des bovins porteurs de T. annulata, elle risquerait alors de devenir vecteur de cette protozoose, comme c'est déjà le cas en Mauritanie (6), aboutissant ainsi à une extension de l'aire de distribution de la theilériose tropicale vers le centre et le sud du pays.

\section{- CONCLUSION}

La theilériose tropicale bovine est une infection transmise par les tiques qui fait intervenir trois acteurs, le bovin (hôte intermédiaire), la tique (hôte définitif) et le parasite (Theileria annulata), qui appartiennent à trois groupes très éloignés sur le plan taxonomique. Le bovin se trouve soumis à des conditions d'élevage très différentes d'une région à une autre. La tique vectrice $\mathrm{H}$. $\mathrm{scu}$ pense a une dynamique d'activité intimement liée aux conditions abiotiques. Le protozoaire est soumis à une pression de sélection d'origine immunitaire plus ou moins importante en fonction de l'espèce du boviné et de sa race, variant même avec les individus. Il résulte de toutes ces interactions une typologie épidémiologique à la fois variable dans le temps et dans l'espace, mais aussi compliquée. La connaissance de ces types épidémiologiques est capitale sur deux plans : (a) sur le plan fondamental car il s'agit d'un modèle d'étude de la relation acarien-mammifère-protozoaire, et (b) sur le plan de la lutte contre l'infection par T. annulata car la réussite de la lutte est conditionnée par la disponibilité de données épidémiologiques régionales, locales, voire relatives à l'élevage concerné.

\section{Remerciements}

Cette étude a reçu le soutien du laboratoire «Epidémiologie des infections enzootiques des herbivores en Tunisie : application à la lutte » (ministère de l'Enseignement supérieur et de la Recherche scientifique) et en partie du projet Deutsche Forschungsgemeinschaft «Molecular epidemiology network for promotion and support of delivery of life vaccines against Theileria parva and Theileria annulata infection in Eastern and Northern Africa »(AH 41/7-1).

\section{BIBLIOGRAPHIE}

1. BAHRI S., KALLEL A., GOUIA A., 1995. La theilériose bovine en Tunisie, enquête rétrospective sur cinq années. Bull. Epidémiol. Inform. Vét. Instit. Rech. Vét. Tunis., $5: 1-3$.

2. BEN MILED L., DELLAGI K., BERNARDI G., MELROSE T.R., DARGHOUTH M., BOUATTOUR A., KINNAIRD J., SHIELS B., TAIT A., BROWN C.G., 1994. Genomic and phenotypic diversity of Tunisian Theileria annulata isolates. Parasitology, 108: 51-60.

3. BOUATTOUR A., 1996. Etude des tiques des bovins dans la région de Sidi Thabet : leur rôle dans la transmission de la theilériose. Diplôme études approf. écol. anim., Faculté des Sciences de Tunis, Tunisie, 62 p.

4. BOUATTOUR A., 2001. Les tiques en Tunisie : rôle de Hyalomma detritum dans la transmission de Theileria annulata. Thèse Doct., Faculté des Sciences de Tunis, Tunisie, $247 \mathrm{p}$.

5. BOUATTOUR A., DARGHOUTH M.A., BeN MILED L., 1996. Cattle infestation by Hyalomma detritum ticks and prevalence of Theileria in Hyalomma detritum species in Tunisia. Vet. Parasitol., 65: 256-263.

6. D'OLIVEIRA C., VAN DER WEIDE M., JACQUIET P., JONGEJAN F., 1997. Detection of Theileria annulata by the PCR in ticks (Acari: Ixodidae) collected from cattle in Mauritania. Exp. Appl. Acarol., 21: 279-291

7. DARGHOUTH M.A., BOUATTOUR A., BEN MILED L., KILANI M., BROWN C.G.D., 1996. Epidemiology of tropical theileriosis (Theileria annulata infection of cattle) in an endemic region of Tunisia: characterisation of endemicity states. Vet. Parasitol., 65: 199-211.

8. DARGHOUTH M.A., BOUATTOUR A., BEN MILED L., SASSI L., 1996. Diagnosis of Theileria annulata infection of cattle in Tunisia: comparison of serology and blood smears. Vet. Res., 27: 613-621.

9. DARGHOUTH M.A., BOUATTOUR A., KILANI M., 1999. Tropical theileriosis in Tunisia: epidemiology and control. Parassitologia, 41 (suppl. 1): 33-36.

10. DARGHOUTH M.A., BOUATTOUR A., KILANI M., PRESTON P.M., 2010. Tropical theileriosis (Theileria annulata infection of cattle). In: Lefèvre P.-C., Blancou J., Chermette R., Uilenberg G., éds, Infectious and parasitic diseases of livestock. Paris, France, Lavoisier Tec \& Doc, p. 1841-1855.

11. FATNASSI N., 2010. Etude rétrospective de la dynamique d'apparition des cas de theilériose tropicale dans les gouvernorats de Bizerte, Ariana et Manouba : relation avec la température et I'hygrométrie. Thèse Doct. Méd. Vét., Ecole nationale de médecine vétérinaire, Sidi Thabet, Tunisie, $63 \mathrm{p}$.

12. GHARBI M., SASSI L., DORCHIES P., DARGHOUTH M.A., 2006. Infection of calves with Theileria annulata in Tunisia: economic analysis and evaluation of the potential benefit of vaccination. Vet. Parasitol., 137: 231-241.

13. GHARBI M., TOUAY A., KHAYECHE M., LAARIF J., JEDIDI M., SASSI L., DARGHOUTH M.A., 2011. Ranking control options for tropical theileriosis in at-risk dairy cattle in Tunisia, using benefit-cost analysis. Rev. Sci. Tech. Off. Int. Epizoot., 30: 763-778.

14. GHARBI M., MHADHBI M., DARGHOUTH M.A., 2012. Diagnostic de la theilériose tropicale du bœuf (infection par Theileria annulata) en Afrique du Nord. Rev. Méd. Vét., 12 : 563-571.

15. GHARBI M., HAYOUNI M.E., SASSI L., DRIDI O., DARGHOUTH M.A., 2013. Hyalomma scupense (Acari, Ixodidae) in Northeast Tunisia: seasonal population dynamics of nymphs and adults on field cattle. Parasite, 20: 12. DOI: 10.1051/parasite/2013012

16. GHARBI M., MOUSSI N., JEDIDI M., MHADHBI M., SASSI L., DARGHOUTH M.A., 2013. Population dynamics of ticks infesting the one-humped camel (Camelus dromedarius) in Central Tunisia. Ticks TickBorne Dis., 4: 488-491.

17. GLASS E.J., 2001. The balance between protective immunity and pathogenesis in tropical theileriosis: what we need to know to design effective vaccines for the future. Res. Vet. Sci., 70: 71-75.

18. GLASS E.J., PRESTON P.M., SPRINGBETT A., CRAIGMILE S., KIRVAR E., WILKIE G., BROWN C.G.D., 2005. Bos taurus and Bos indicus (Sahiwal) calves respond differently to infection with Theileria annulata and produce markedly different levels of acute phase proteins. Int. J. Parasitol., 35: 337-347. 
19. GOSRANI E., 1999. Contribution à l'étude épidémiologique d'un foyer de la theilériose bovine à Theileria annulata dans I'oasis de Gabès. Thèse Doct. Méd. Vét., Ecole nationale de médecine vétérinaire, Sidi Thabet, Tunisie, $72 \mathrm{p}$.

20. KAROUI M., 2002. Suivi de l'infection sur les veaux en première saison de theilériose tropicale dans l'état d'endémie stable : application à l'évaluation de l'intérêt de la vaccination contre la theilériose tropicale. Thèse Doct. Méd. Vét., Ecole nationale de médecine vétérinaire, Sidi Thabet, Tunisie, $68 \mathrm{p}$.

21. KHAYECHE M., 1998. Essai de caractérisation des situations endémiques génératrices de cas cliniques de theilériose bovine à Theileria annulata dans la région de Medjerda. Thèse Doct. Méd. Vét., Ecole nationale de médecine vétérinaire, Sidi Thabet, Tunisie, 58 p.

22. LEBBI R., 1991. Contribution à l'étude épidémiologique de la theilériose bovine dans la région de Sidi Thabet. Etude comparée du parasitisme des veaux adultes par les tiques Hyalomma. Thèse Doct. Méd. Vét., Ecole nationale de médecine vétérinaire, Sidi Thabet, Tunisie, 62 p.

23. MCHARDY N., WEKESA L.S., HUDSON A.T., RANDALL A.W., 1985. Antitheilerial activity of BW720C (buparvaquone): a comparison with parvaquone. Res. Vet. Sci., 39: 29-33.

24. MHADHBI M., NAOUACH A., BOUMIZA A., CHAABANI M.F., BENABDERAZZAK S., DARGHOUTH M.A., 2010. In vivo evidence for the resistance of Theileria annulata to buparvaquone. Vet. Parasitol., 169: 241-247.

25. MAHMMOD Y.S., ELBALKEMY F.A., KLAAS I.C., ELMEKKAWY M.F., MONAZIE A.M., 2011. Clinical and haematological study on water buffaloes (Bubalus bubalis) and cross-bred cattle naturally infected with Theileria annulata in Sharkia province, Egypt. Ticks Tick-Borne Dis., 2: 168-171.

26. MAHONEY D.F., 1977. Babesia of domestic animals. In: Kreier J.P. Ed., Parasitic protozoa, Vol. IV. New York, USA, Academic Press, p. 1-52.

27. MINISTERE DE L'AGRICULTURE, 2006. Enquête sur les structures des exploitations agricoles. Tunis, Tunisie, ministère de l'Agriculture, $136 \mathrm{p}$.

\section{Summary}

Gharbi M., Rjeibi M.R., Darghouth M.A. Epidemiology of tropical bovine theileriosis (Theileria annulata infection) in Tunisia: A review

This article reviews the literature on the epidemiology of tropical theileriosis in Tunisia. It is a specific parasitic disease caused by the presence and proliferation in mononuclear phagocytes, then in erythrocytes of a protozoan belonging to the family Theileridae, Theileria annulata. It is biologically transmitted by several species of ixodid ticks belonging to the genus Hyalomma. The three actors involved have a very different taxonomy causing a disease whose epidemiology is particularly complex. This infection consists of three enzootic ways: enzootic stability due to an equilibrium between the host and the parasite, (ii) low enzootic instability due to the presence of a low tick population causing clinical cases in animals aged 2 to 3 years, and (iii) high enzootic instability in which the tick population is so low that the probability of a contact between an infected tick and a susceptible host is very low. Depending on the epidemiological situation, the farms can plan adapted control programs.

Keywords: Cattle - Bovine tropical theileriosis - Epidemiology Disease control - Tunisia.
28. MINJAW B., MCLEOD A., 2003. Tick-borne diseases and poverty: The impact of ticks and tick-borne diseases on the livelihoods of smallscale and marginal livestock owners in India and Eastern and Southern Africa. Research report. University of Edinburgh, UK, Centre of Tropical Veterinary Medicine, $116 p$

29. SALIH D.A., SHARIEFF O.E., LAZARUS A.G, HASSAN S.M, EL HUSSEIN A.M., 2005. Natural infection rates and transmission of Theileria annulata by Hyalomma anatolicum anatolicum ticks in the Sudan. Onderstepoort J. Vet. Res., 72: 303-307.

30. SERGENT E., DONATIEN A., PARROT L., LESTOQUARD F., 1945. Etude des piroplasmoses bovines. Alger, Algérie, Institut Pasteur, $816 \mathrm{p}$.

31. SOUDANI M.C., 1995. Contribution à l'étude épidémiologique de la theilériose bovine à Theileria annulata : analyse clinique, parasitologique et sérologique de l'infection naturelle des veaux en première saison estivale. Thèse Doct. Méd. Vét., Ecole nationale de médecine vétérinaire, Sidi Thabet, Tunisie, $68 \mathrm{p}$

32. TOUHAMI C., 2012. Contribution à l'étude de l'incidence des hémopathogènes des bovins au nord de la Tunisie. Thèse Doct. Méd. Vét., Ecole nationale de médecine vétérinaire, Sidi Thabet, Tunisie, $103 \mathrm{p}$.

33. VISERAS J., HUELI L.E., ADROHER F.J., GARCIA-FERNANDEZ P., 1999. Studies on the transmission of Theileria annulata to cattle by the tick Hyalomma lusitanicum. J. Vet. Med. B, 46: 505-509.

34. WALKER A.R., BOUATTOUR A., CAMICAS J.-L., ESTRADA-PENA A., HORAK I.G., LATIF A.A., PEGRAM R.G., PRESTON P.M., 2003. Ticks of domestic animals in Africa: a guide to identification of species. Edinburgh, UK, Bioscience Reports.

35. WEIR W., KARAGENC T., GHARBI M., SIMUUNZA M., AYPAK S. AYSUL N., DARGHOUTH M.A., SHIELS B., TAIT A., 2011. Population diversity and multiplicity of infection in Theileria annulata. Int. J. Parasitol., 41: 193-203.

Accepted 2 February 2015; Online publication 30 September 2015

\section{Resumen}

Gharbi M., Rjeibi M.R., Darghouth M.A. Epidemiología de la theileriosis tropical bovina (infección por Theileria annulata) en Túnez: síntesis

El presente artículo es una revisión bibliográfica de la epidemiologia de la teilerosis tropical bovina en Túnez. Es una parasitosis específica debida a la presencia y a la multiplicación en los fagocitos mononucleados y luego en los eritrocitos de un protozoario de la familia Theileridae, Theileria annulata. Se transmite de manera biológica, mediante varias especies de garrapatas de la familia Ixodidae, pertenecientes al género Hyalomma. La implicación de tres actores muy alejados sobre el plan taxonómico se encuentra al origen de la enfermedad, cuya epidemiología es compleja. Esta infección evoluciona según tres formas enzooticas: a) la enzootia estable que resulta de un estado de equilibrio entre el huésped y el parásito, b) la enzootia inestable moderada debida a la presencia de una baja población de garrapatas generando casos clínicos en los animales de dos a tres años de edad y c) la enzootia inestable elevada en la cual la población de garrapatas es tan baja que la probabilidad de encuentro entre la garrapata infectada y el huésped sensible es mínima. El tipo de situación epidemiológica en el que se encuentra el criadero permite planificar un programa de lucha adaptado.

Palabras clave: Ganado bovino - Theileriosis tropical bovina Epidemiología - Control de enfermedades - Túnez. 
